\title{
Laparoscopic repeated pancreatectomy for isolated local recurrence in remnant pancreas following laparoscopic radical pancreatectomy for pancreatic ductal adenocarcinoma: Two cases report
}

\author{
Munseok Choi ${ }^{1,2,3}$, Suk Jun Lee ${ }^{2}$, Dong-Min Shin ${ }^{2}$, Ho Kyoung Hwang ${ }^{1,2,3}$, \\ Woo Jung Lee ${ }^{1,2,3}$, and Chang Moo Kang ${ }^{1,2,3}$ \\ ${ }^{1}$ Division of Hepatobiliary and Pancreatic Surgery, ${ }^{2}$ Department of Surgery, Yonsei University College of Medicine, \\ ${ }^{3}$ Pancreatobiliary Cancer Center, Yonsei Cancer Center, Severance Hospital, Seoul, Korea \\ Typical treatment for recurrent pancreatic cancer is potent chemotherapy or chemoradiotherapy. However, recent clin- \\ ical investigations suggest a potential therapeutic role of local resection in recurrent pancreatic cancer. Based on accu- \\ mulating surgical experience of minimally invasive pancreatectomy, we report cases of two patients who underwent \\ successful laparoscopic re-resection of isolated local recurrence following laparoscopic radical pancreatectomy for pan- \\ creatic cancer. (Ann Hepatobiliary Pancreat Surg 2020;24:542-546)
}

Key Words: Laparoscopy; Pancreatic neoplasms; Pancreatectomy; Neoplasm recurrence; Local

\section{INTRODUCTION}

Recurrent pancreatic cancer is generally treated with potent chemotherapy or chemoradiotherapy. However, recent clinical investigations suggest a potential oncologic role of local resection in recurrent pancreatic cancer. Groot et al. ${ }^{1}$ performed a systematic review on isolated local recurrence of pancreatic cancer. Based on eight published studies, including 100 patients who underwent re-resection of recurrent pancreatic cancer, they concluded that local re-resection of recurrent pancreatic could be feasible, safe, and effective in selected patients. They demonstrated that postoperative morbidity and mortality were $29 \%$ and $1 \%$, respectively. Median survival was much higher (32 months) than other treatment modalities, such as chemotherapy (19 months) or radiotherapy (16 months).

Based on accumulating surgical experience, laparoscopic distal pancreatectomy ${ }^{2,3}$ and laparoscopic pancreaticoduodenectomy ${ }^{4,5}$ are regarded as safe potential options in well-selected pancreatic cancer patients. Herein, we report two patients who underwent successful repeat laparoscopic pancreatectomy for isolated local recurrence following laparoscopic radical pancreatectomy for pancreatic cancer.

\section{CASE}

\section{Case 1}

A 68-year-old Korean man was found to have recurrent pancreatic cancer on follow-up computed tomography (CT). He had a medical history of hypertension and diabetes, and was a hepatitis $\mathrm{C}$ virus carrier. He also underwent laparoscopic small bowel segmental resection for a gastrointestinal stromal tumor (ileum) in 2012, as well as right posterior sectionectomy for hepatocellular carcinoma in 2015. During regular follow-up, pancreatic tail cancer (pT3N0) was incidentally detected, and he underwent laparoscopic radical distal pancreatectomy with splenectomy on June 7, 2016. The pathologic result was ductal adenocarcinoma, well-differentiated (pT3N0M0, stage IIA).

Received: May 18, 2020; Revised: June 28, 2020; Accepted: July 9, 2020

Corresponding author: Chang Moo Kang

Department of Surgery, Yonsei University College of Medicine, Ludlow Faculty Research Building \#201, 50 Yonsei-ro, Seodaemun-gu, Seoul 03722, Korea

Tel: +82-2-2228-2135, Fax: +82-2-313-8289, E-mail: cmkang@yuhs.ac

Copyright (C) 2020 by The Korean Association of Hepato-Biliary-Pancreatic Surgery

This is an Open Access article distributed under the terms of the Creative Commons Attribution Non-Commercial License (http://creativecommons.org/ censes/by-nc/4.0) which permits unrestricted non-commercial use, distribution, and reproduction in any medium, provided the original work is properly cited. Annals of Hepato-Biliary-Pancreatic Surgery - pISSN: 2508-5778 - elSSN: 2508-5859 
The patient received six cycles of gemcitabine as adjuvant chemotherapy from July 4 to December 19, 2016.

Physical examination revealed no specific findings. Tumor marker CA 19-9 was elevated (217.0 U/ml). Follow-up CT (April 23, 2018) showed a 2.3-cm low attenuating lesion at the pancreas resection margin, suggesting local tumor recurrence. Further evaluation with PET-CT suggested no metastatic lesions; therefore, the surgical option for local recurrence was selected over chemotherapy.

The patient underwent laparoscopic segmental resection of the pancreatic neck on May 23, 2018. There were mild to moderate adhesions. Recurrent tumor abutted the posterior wall of the stomach, suggesting cancer invasion. Gastric wedge resection was done by endo-GIA in order to achieve en-bloc resection of the cancer. Finally, the proximal portion of the remnant splenic artery was dissected and resected by endo-GIA (Fig. 1).

The tumor was recurrent pancreatic ductal adenocarcinoma with tumor size of $2.4 \mathrm{~cm}$ in maximum diameter. Lymphovascular invasion was noted without perineural invasion. All resection margins, including pancreatic duct and circumferential margin, were negative for carcinoma cells. In total, eight lymph nodes were retrieved and all were negative. The patient was discharged seven days postoperatively. The oncology department gave the patient 13 cycles of gemcitabine and abraxane as postoperative adjuvant chemotherapy. Chemotherapy was stopped on November 8, 2018, due to acute kidney injury. There were no definite metastatic lesions in the abdomen on follow-up CT 1 year after surgery.

\section{Case 2}

A 65-year-old Korean woman was admitted to the hospital due to isolated local recurrence in the remnant pancreas. She previously underwent laparoscopic pancreaticoduodenectomy with combined segmental resection of the SMV-SV-PV confluence on April 4, 2018. The patient received postoperative adjuvant chemotherapy for six months with a gemcitabine regimen. During her regular follow-up, local recurrence near the previous pancreaticojejunostomy site was detected. Follow-up CT with pancreas dynamic spiral 3D showed a new suspicious mass at the pancreaticojejunostomy site. Preoperative tumor markers (CEA, $8.66 \mathrm{ng} / \mathrm{ml}$, and CA19-9, $35.2 \mathrm{U} / \mathrm{ml}$ ) were checked. No further progression was noted during adjuvant chemotherapy. Therefore, she decided to undergo laparoscopic completion total pancreatectomy on May 29, 2019. As a result of the previous laparoscopic approach, there were moderate adhesions in the abdominal cavity and around the pancreaticojejunostomy sites.

To facilitate the surgical procedure, the pancreaticosplenic ligament was divided using endo-GIA. The jejunum was dissected and divided near the previous hepaticojejunostomy site. Remnant pancreas was dissected, but it was challenging to dissect from the superior mesenteric artery and the splenic artery due to severe inflammatory changes and tumor infiltration. Vascular en-
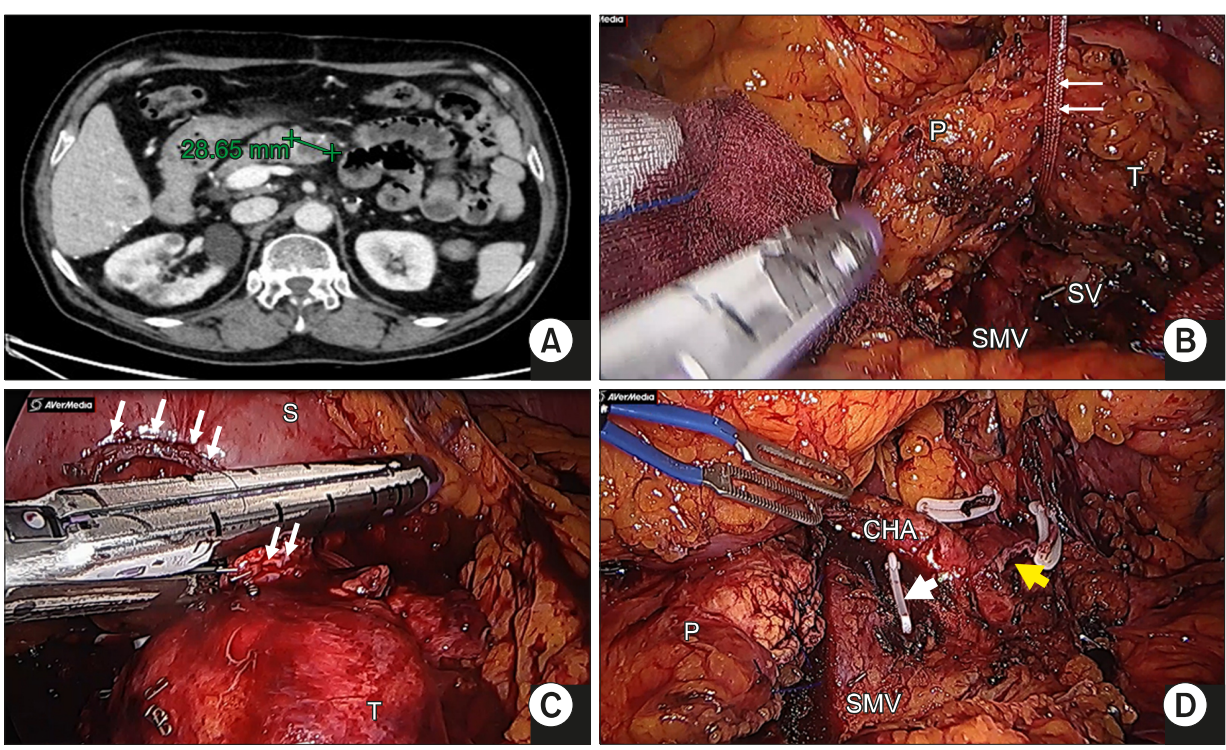

Fig. 1. Perioperative findings. Recurrent pancreatic cancer was noted on remnant pancreas (A). The pancreatic neck was fully mobilized from the SMV-PVSV confluence (B). Combined gastric wedge resection (thin white arrow) was performed (C). The splenic artery was dissected and resected by endo-GIA (yellow arrow), and the splenic vein was also ligated (thick arrow, D). T, tumor area in remnant pancreas; $\mathrm{P}$, pancreatic neck; SMV, superior mesenteric vein; SV, splenic vein; S, stomach; CHA, common hepatic artery. 

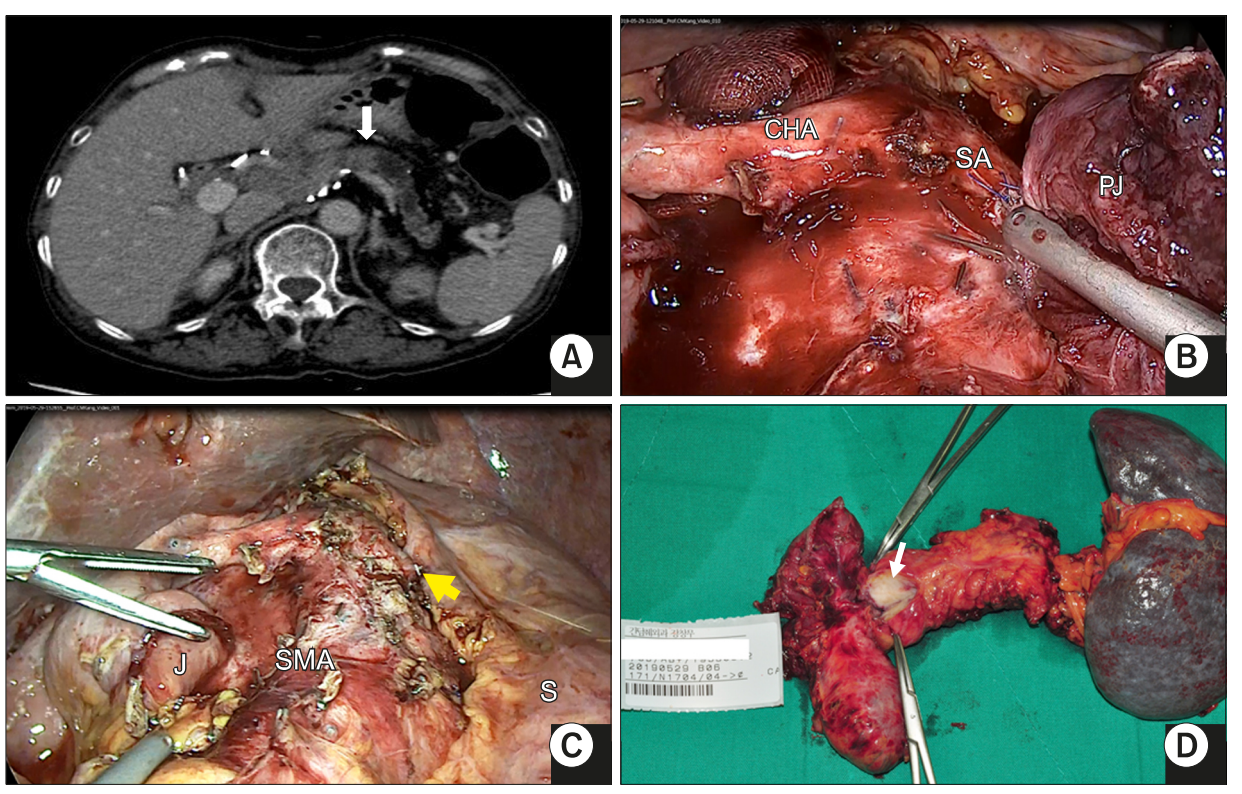

Fig. 2. Perioperative findings. Recurrent pancreatic cancer at the pancreaticojejunostomy (PJ) site (white arrow, A). The jejunum was resected near PJ site (B). Tumor was dissected from the superior mesenteric artery. The splenic artery was ligated with vascular endo GIA (yellow arrow, C). Gross findings of recurrent tumor at the PJ site (white arrow, D). CHA, common hepatic artery; SA, splenic artery; PJ, previous pancreaticojejunostomy site; $\mathrm{J}$, proximal jejunum after resecting previous Roux-en-Y limb for PJ; SMA, superior mesenteric artery.
do-GIA was applied to control the splenic artery. The total operation time was $270 \mathrm{~min}$, and the estimated blood loss was $650 \mathrm{ml}$. Pathological examination revealed recurrent adenocarcinoma involving the pancreas and small bowel. The tumor size was $1.7 \times 1.5 \mathrm{~cm}$. Lymphovascular invasion was noted without perineural invasion. The posterior margin of the pancreas showed extension of the carcinoma. Other resection margins, including the anterior pancreas, lateral small bowel, and spleen, were free from carcinoma cells. The patient was discharged 12 days postoperatively. Radiotherapy and adjuvant chemotherapy were planned (Fig. 2).

\section{DISCUSSION}

Regarding long-term oncologic outcomes of repeat pancreatectomy for isolated local recurrence of pancreatic cancer, Yamada et al. ${ }^{6}$ reported 114 patients with remnant pancreatic cancer after initial pancreatectomy. A total of 90 patients underwent repeat pancreatectomy and median survival was 26 months, superior to that of non-resected patients (HR: 0.56, $p=0.012$ ). Hashimoto et al. ${ }^{7}$ reviewed 12 published studies about recurrent pancreatic cancer in remnant pancreas following initial pancreatectomy, showing overall survival after repeat pancreatectomy for remnant pancreatic cancer of 14 to 35.5 months, which was longer than that of unresectable pancreatic cancer patients in recent studies. ${ }^{8,9}$ In addition, Zhou et al. ${ }^{10}$ performed pooled analysis of the second pancreatectomy for recurrent pancreatic ductal adenocarcinoma. They retrieved 55 patients from 19 published articles and investigated long-term overall survival. It was found that 1-, 3-, and 5 -year overall survival rates after repeat pancreatectomy were $82.2 \%, 49.2 \%$, and $40.6 \%$, respectively, suggesting a potential role of repeat pancreatectomy for isolated recurrent pancreatic cancer in remnant pancreas.

Only a few cases of laparoscopic repeat pancreatectomy have been published, and only two reported the technical feasibility of laparoscopic repeat pancreatectomy after initial pancreatectomy (Table 1). Sunagawa et al. ${ }^{11}$ reported the first case successfully treated by laparoscopic completion total pancreatectomy for newly developed pancreatic cancer in remnant pancreas after laparoscopic pancreaticoduodenectomy for distal bile duct cancer. Recently, Nakagawa et al. ${ }^{12}$ also published a case of laparoscopic pancreaticoduodenectomy for remnant pancreatic recurrence after laparoscopic distal pancreatectomy and hepatectomy for leiomyosarcoma. In this report, pancreaticojejunostomy was performed through a small epigastric wound where the surgical specimen was removed.

To the best of our knowledge, the present two cases are the first to show the technical feasibility and safety of laparoscopic repeat pancreatectomy for recurrent pancreatic ductal adenocarcinoma in remnant pancreas after initial laparoscopic radical pancreatectomy for primary pancreatic cancer. Minimally invasive pancreatectomy in 
Table 1. Cases reporting technical feasibility of laparoscopic repeated pancreatectomy

\begin{tabular}{|c|c|c|c|c|c|c|c|c|c|c|c|c|}
\hline Author, Year & $\mathrm{N}$ & Age & Sex & $\begin{array}{l}\text { Diag- } \\
\text { nosis }\end{array}$ & 1 st OP & $\begin{array}{l}\text { Time } \\
\text { interval }\end{array}$ & 2nd OP & $\mathrm{EBL}, \mathrm{ml}$ & $\begin{array}{l}\text { OT, } \\
\text { min }\end{array}$ & LOH & $\begin{array}{l}\text { Compli- } \\
\text { cation }\end{array}$ & Survival, months \\
\hline $\begin{array}{l}\text { Sunakawa et al., } \\
2014\end{array}$ & 1 & 72 & $\mathrm{~F}$ & $\mathrm{BDC}$ & PPPD & 18 & CTP & 1200 & 462 & 14 & No & NA \\
\hline $\begin{array}{l}\text { Nagakawa et al., } \\
2019\end{array}$ & 1 & 67 & $\mathrm{~F}$ & LMS & DPS & 10 & SSPPD & 50 & 274 & 13 & No & 16 months, alive \\
\hline \multirow[t]{2}{*}{ Present, 2019} & 1 & 68 & M & $\mathrm{PC}$ & DPS & 23 & $\mathrm{P}$ & 200 & 290 & 7 & No & 22, alive, NED \\
\hline & 2 & 65 & $\mathrm{~F}$ & $\mathrm{PC}$ & PPPD & 11 & CTP & 650 & 270 & 12 & No & 10 months, alive, NED \\
\hline
\end{tabular}

BDC, bile duct cancer; LMS, leiomyosarcoma; PC, pancreatic cancer; PPPD, pylorus preserving pancreaticoduodenectomy; SSPPD, subtotal stomach-preserving pancreaticoduodenectomy; PR, partial pancreatic resection; CTP, completion total pancreatectomy; NED, no evidence of disease; NA, missing value; EBL, estimated blood loss; OP, operation; OT, operation time; $\mathrm{LOH}$, length of hospital stay

pancreatic cancer remains controversial. However, our recent experiences ${ }^{3,5}$ and meta-analysis ${ }^{2,4}$ suggest the technical and oncological safety of a minimally invasive approach to well-selected pancreatic cancer.

These are our rationales for minimally invasive radical pancreatectomy for pancreatic cancer: 1) Laparoscopic margin-negative resection without ruining oncologic principle is quite feasible. 2) Usually, pancreatic head cancer is associated with a hard pancreas with a relatively large pancreatic duct, which can be related to a rare incidence of postoperative pancreatic fistula. 3) Postoperative patient recovery appears to be quite smooth due to the benefit of minimally invasive surgery, 4) which can provide good clinical conditions for subsequent postoperative adjuvant chemotherapy. 5) Finally, postoperative adhesions are quite limited, which is appropriate for unexpected reoperation, including repeat pancreatectomy.

Further research is needed to establish the indications for minimally invasive pancreatectomy for pancreatic cancer. In addition, well-designed clinical investigations and an expert consensus are needed for developing management guidelines toward minimally invasive radical pancreatectomy for resectable pancreatic cancer and repeat pancreatectomy for isolated local recurrence of pancreatic cancer in remnant pancreas.

\section{CONFLICT OF INTEREST}

No potential conflict of interest relevant to this article was reported.

\section{ORCID}

Munseok Choi: https://orcid.org/0000-0002-9844-4747

Suk Jun Lee: https://orcid.org/0000-0003-3839-5071

Dong-Min Shin: https://orcid.org/0000-0002-8802-5370

Ho Kyoung Hwang: https://orcid.org/0000-0003-4064-7776

Woo Jung Lee: https://orcid.org/0000-0001-9273-261X

Chang Moo Kang: https://orcid.org/0000-0002-5382-4658

\section{AUTHOR CONTRIBUTIONS}

Writing - Original Draft: MC. Revised the manuscript: SJL, DMS. Supervision: HKH, WJL. Conceptualization, Writing - Review \& Editing, Validation: CMK.

\section{REFERENCES}

1. Groot VP, van Santvoort HC, Rombouts SJ, Hagendoorn J, Borel Rinkes IH, van Vulpen M, et al. Systematic review on the treatment of isolated local recurrence of pancreatic cancer after surgery; re-resection, chemoradiotherapy and SBRT. HPB (Oxford) 2017;19:83-92.

2. Ricci C, Casadei R, Taffurelli G, Toscano F, Pacilio CA, Bogoni $\mathrm{S}$, et al. Laparoscopic versus open distal pancreatectomy for ductal adenocarcinoma: a systematic review and meta-analysis. J Gastrointest Surg 2015;19:770-781.

3. Lee SH, Kang CM, Hwang HK, Choi SH, Lee WJ, Chi HS. Minimally invasive RAMPS in well-selected left-sided pancreatic cancer within Yonsei criteria: long-term (>median 3 years) oncologic outcomes. Surg Endosc 2014;28:2848-2855.

4. Peng L, Zhou Z, Cao Z, Wu W, Xiao W, Cao J. Long-term oncological outcomes in laparoscopic versus open pancreaticoduodenectomy for pancreatic cancer: a systematic review and meta-analysis. J Laparoendosc Adv Surg Tech A 2019;29:759769.

5. Choi M, Hwang HK, Rho SY, Lee WJ, Kang CM. Comparing laparoscopic and open pancreaticoduodenectomy in patients with pancreatic head cancer: oncologic outcomes and inflammatory scores. J Hepatobiliary Pancreat Sci 2020;27:124-131. 
6. Yamada S, Kobayashi A, Nakamori S, Baba H, Yamamoto M, Yamaue $\mathrm{H}$, et al. Resection for recurrent pancreatic cancer in the remnant pancreas after pancreatectomy is clinically promising: results of a project study for pancreatic surgery by the Japanese Society of Hepato-Biliary-Pancreatic Surgery. Surgery 2018;164:1049-1056.

7. Hashimoto D, Chikamoto A, Masuda T, Nakagawa S, Imai K, Yamashita YI, et al. Pancreatic cancer arising from the remnant pancreas: is it a local recurrence or new primary lesion? Pancreas 2017;46:1083-1090.

8. Conroy T, Desseigne F, Ychou M, Bouché O, Guimbaud R, Bécouarn Y, et al. FOLFIRINOX versus gemcitabine for metastatic pancreatic cancer. N Engl J Med 2011;364:1817-1825.

9. Lee K, Bang K, Yoo C, Hwang I, Jeong JH, Chang HM, et al.
Clinical outcomes of second-line chemotherapy after progression on nab-paclitaxel plus gemcitabine in patients with metastatic pancreatic adenocarcinoma. Cancer Res Treat 2020;52:254-262.

10. Zhou Y, Song A, Wu L, Si X, Li Y. Second pancreatectomy for recurrent pancreatic ductal adenocarcinoma in the remnant pancreas: a pooled analysis. Pancreatology 2016;16:1124-1128.

11. Sunagawa H, Mayama Y, Orokawa T, Oshiro N. Laparoscopic total remnant pancreatectomy after laparoscopic pancreaticoduodenectomy. Asian J Endosc Surg 2014;7:71-74.

12. Nakagawa Y, Fukami Y, Harada T, Maeda A, Takayama Y, Takahashi $\mathrm{T}$, et al. Laparoscopic pancreaticoduodenectomy for remnant pancreatic recurrence after laparoscopic distal pancreatectomy and hepatectomy for greater omentum leiomyosarcoma. Asian J Endosc Surg 2020;13:117-120. 